\title{
Synapse-Associated Protein-97 Isoform-Specific Regulation of Surface AMPA Receptors and Synaptic Function in Cultured Neurons
}

\author{
Gavin Rumbaugh, ${ }^{1}$ Gek-Ming Sia, ${ }^{1}$ Craig C. Garner, ${ }^{2}$ and Richard L. Huganir ${ }^{1}$ \\ ${ }^{1}$ Howard Hughes Medical Institute and Department of Neuroscience, The Johns Hopkins University School of Medicine, Baltimore, Maryland 21205, and \\ the 2Department of Psychiatry and Behavioral Science, Stanford University, Palo Alto, California 94304-5485
}

\begin{abstract}
Members of the synapse-associated protein-97 (SAP97) family of scaffold proteins have been implicated as central organizers of synaptic junctions to build macromolecular signaling complexes around specific postsynaptic neurotransmitter receptors. In this regard, SAP97 has been suggested to regulate the synaptic localization of glutamate receptor type 1 subunits of the AMPA-type glutamate receptors. To test this hypothesis directly, we assessed the effects of SAP97 overexpression on surface expression of synaptic AMPA receptors. We find that recombinant SAP97 not only becomes concentrated at synaptic junctions but also leads to an increase in synaptic AMPA receptors, spine enlargement, and an increase in miniature EPSC (mEPSC) frequency, indicating that SAP97 has both postsynaptic and presynaptic effects on synaptic transmission. Synaptic targeting of SAP97, increased surface AMPA receptors, and increased mEPSC frequency are dependent on the presence of specific alternatively spliced sequences in SAP97 that encode a protein 4.1 binding site. These results suggest that SAP97 can affect the synaptic recruitment of AMPA receptors and spine morphology and that these effects may be regulated by alternative splicing.
\end{abstract}

Key words: synaptic transmission; MAGUKs; SAPs; trafficking; mEPSC; glutamate

\section{Introduction}

Excitatory transmission in the cortex and hippocampus usually occurs at specialized structures called dendritic spines. Spines contain an electron-dense web of proteins called the postsynaptic density (PSD). Within the PSD, there are a variety of receptors, such as AMPA- and NMDA-type glutamate receptors, and an array of intracellular proteins that mediate organization of molecules critical for proper synaptic transmission. Synapses are highly plastic, and recent evidence suggests that AMPA receptors (AMPARs) are added or removed from individual synapses on the basis of their previous history of (Shi et al., 1999; Hayashi et al., 2000). Therefore, molecules involved in trafficking and organization of glutamate receptors are probably key modulators of synaptic transmission.

Recently, there has been interest in the synapse-associated proteins (SAPs) because of their presence at excitatory synapses and their ability to bind to membrane receptors, signaling molecules, and the cytoskeleton (Garner et al., 2000). This family of proteins consist of four mammalian homologues: SAP90/PSD95 (Cho et al., 1992; Kistner et al., 1993), SAP97/hDLG (Lue et al., 1994; Muller et al., 1995), PSD93/Chapsyn-110 (Brenman et al., 1996; Kim et al., 1996), and SAP102 (Muller et al., 1996). These proteins are roughly $70 \%$ homologous to each other, and all con-

Received Feb. 5, 2002; revised March 21, 2003; accepted March 24, 2003.

This work was supported by the Howard Hughes Medical Institute (R.L.H.) and National Research Scholar Award F32 NS43071-01 (G.R.). We thank Dr. David Linden and Dr. Gareth Thomas for their helpful comments in the preparation of this manuscript.

Correspondence should be addressed to Dr. Richard L. Huganir, Johns Hopkins University School of Medicine, Department of Neuroscience and Howard Hughes Medical Institute, 725 North Wolfe Street, PCTB 904, Baltimore, MD 21205. E-mail: rhuganir@jhmi.edu.

Copyright $\odot 2003$ Society for Neuroscience $\quad$ 0270-6474/03/234567-10\$15.00/0 tain three PDZ domains, a single SH3 domain, a HOOK domain, and an inactive guanylate kinase (GK) domain. SAP family proteins have been found to bind to AMPA, NMDA, and kainate receptors at synapses (Garner et al., 2000) and signaling molecules (Kim et al., 1998; Colledge et al., 2000) and other transmembrane proteins (Chen et al., 2000). Because of the diverse proteins that SAPs can associate with at synapses, these molecules are thought to act as scaffolds that organize the PSD.

In neurons, SAP97 has been found to be present in both axons (Muller et al., 1995) and postsynaptic densities of excitatory synapses (Valtschanoff et al., 2000; Aoki et al., 2001). SAP97 has been shown to bind to the glutamate receptor type 1 (GluR1) subunit of the AMPA receptor, and this interaction has been shown to be PDZ dependent (Leonard et al., 1998; Cai et al., 2002). In addition, SAP97 has been shown to bind to the PKA anchoring molecule AKAP (Colledge et al., 2000) and the actin and GluR1 binding protein 4.1 (Lue et al., 1994; Shen et al., 2000). Moreover, PKA has been implicated in the modulation of AMPA receptors (Banke et al., 2000) and LTP (Lee et al., 2000). Together, these data suggest that SAP97 may be important for proper synaptic function, possibly by modulating AMPA receptor trafficking at the synaptic membrane.

In this study, we describe the subcellular localization of SAP97 in primary dissociated hippocampal neurons and its colocalization with synaptic markers. Transfection of green fluorescent protein (GFP)-tagged SAP97 constructs reveals a preferential targeting of SAP97 to synaptic sites. This synaptic targeting of SAP97 is dependent on an alternatively spliced region between the $\mathrm{SH} 3$ and GK domains called the I3 region, a known protein 4.1 binding site. The synaptic targeting of GFP-SAP97 results in an enlargement of the spine head along with increasing the amount of 
surface GluR-containing AMPA receptors in neurons and an increase in the frequency miniature EPSCs (mEPSCs).

\section{Materials and Methods}

Electrophysiology and mEPSC analysis. Whole-cell patch-clamp recordings were performed from cortical cultures at the day in vitro (DIV) indicated. To isolate AMPA-mediated mEPSCs, neurons were perfused continuously with artificial CSF (aCSF) at a flow rate of $<1 \mathrm{ml} / \mathrm{min}$. The composition of aCSF was as follows (in $\mathrm{mM}$ ): $150 \mathrm{NaCl}, 3.1 \mathrm{KCl}, 2 \mathrm{CaCl}_{2}$, $2 \mathrm{MgCl}_{2}, 10$ HEPES, 0.1 DL-APV, 0.005 strychnine, 0.1 picrotoxin, and 0.001 tetrodotoxin. The osmolarity of the aCSF was adjusted to $305-310$, and $\mathrm{pH}$ was 7.3-7.4. Intracellular saline consisted of (in $\mathrm{mM}$ ): $135 \mathrm{Cs}-$ $\mathrm{MeSO}_{4}, 10 \mathrm{CsCl}, 10 \mathrm{HEPES}, 5$ EGTA, $2 \mathrm{MgCl}_{2}, 4 \mathrm{Na}-\mathrm{ATP}$, and $0.1 \mathrm{Na}-$ GTP. This saline was adjusted to $290-295 \mathrm{mOsm}$, and $\mathrm{pH}$ was 7.2 .

Transfected neurons were selected based on fluorescent (GFP) signal. Once the whole-cell recording configuration was achieved, neurons were voltage-clamped and passive properties were monitored throughout. In the event of a change in series resistance $(R s)$ or input resistance $(R i)$ $>15 \%$ during the course of a recording, the data were excluded from the set. mEPSCs were acquired through an Axopatch 200B amplifier (Axon Instruments, Union City, CA), filtered at $2 \mathrm{kHz}$, and digitized at $5 \mathrm{kHz}$. Sweeps $(10 \mathrm{sec})$ with zero latency were acquired until a sufficient number of events were recorded (a minimum of $5 \mathrm{~min}$ ). Data were recorded continuously only after a period of 1-2 min, during which the cell was allowed to stabilize. mEPSCs were detected manually with MiniAnalysis (Synaptosoft Inc, Decatur, GA) by setting the amplitude threshold to $\sqrt{ }$ RMS $\times 3$ (usually $4 \mathrm{pA}$ ). Once a minimum of 100 events had been collected from a neuron, the amplitude, frequency, rise time (time to peak), decay time (10-90\%), and passive properties were measured. In all electrophysiological experiments, a similar amount of data was acquired from both GFP-SAP97 (and deletions) and GFP-expressing neurons on the same day. Data from each group were then averaged, and statistical significance was determined by Student's $t$ test. All electrophysiological experiments were performed from at least three different platings of neurons from three different transfections.

Immunocytochemistry, microscopy, and data analysis. We generated polyclonal antibodies (pAbs) to SAP97 (JH 4089) by constructing a fusion protein of residues 1-97 of the published rat SAP97 sequence (Muller et al., 1995). Other antibodies used in this study that have been described previously include NR1 [monoclonal antibody (mAb); s3c11], GluR1 (pAb; JH1979) (Liao et al., 1999), PSD-95 (mAb; Upstate, Waltham, MA), Bassoon (mAb; StressGen, Victoria, British Columbia, Canada), BiP (mAb; StressGen), NFH (mAb; Zymed, San Francisco, $\mathrm{CA}$ ), and GFP (3e6, mAb; Molecular Probes, Eugene, OR).

In general, neurons were fixed with a $4 \%$ paraformaldehyde, $4 \%$ sucrose PBS solution for $10 \mathrm{~min}$. Neurons were subsequently permeabilized by $0.2 \%$ Triton X-100 in PBS for 10 min, then blocked for $30-40$ min in $10 \%$ normal donkey serum (NDS). Most primary antibodies were diluted in 10\% NDS and incubated with neurons for $1 \mathrm{hr}$ at room temperature or overnight at $4^{\circ} \mathrm{C}$. FITC- or CY3-conjugated secondary antibodies (Jackson ImmunoResearch, West Grove, PA) to the appropriate species (rabbit or mouse) were diluted in 10\% NDS and incubated at room temperature for $1 \mathrm{hr}$. Coverslips were mounted on precleaned slides with PermaFluor and DABCO and allowed to dry at $37^{\circ} \mathrm{C}$ for $1 \mathrm{hr}$. To label surface GluR1-containing AMPA receptors, we first diluted 10 $\mu \mathrm{g} / \mathrm{ml}$ of CY3-conjugated JH1709 pAb into neuronal growth media and incubated at $37^{\circ} \mathrm{C}$ for $15 \mathrm{~min}$. The unbound excess antibody was quickly washed with fresh warmed growth medium and then fixed and mounted according to the methods described above.

Immunofluorescence was viewed with either an LSM 510 confocal laser scanning microscope or an Axiovert 200 epifluorescence microscope fitted with an Orca ER CCD digital camera (Carl Zeiss, Thornwood, NY). Primary magnification was achieved by either a $63 \times$ PlanApochromat [Carl Zeiss; numerical aperture (NA) 1.4] or $100 \times$ Fluor (Carl Zeiss; NA 1.3) objective. For quantification of surface GluR1 puncta, images were acquired as a two-channel 16 bit binary image. For each neuron expressing GFP-SAP97, both the GFP channel and the GluR1 channel were acquired. We used narrow-band filters for each channel, and no detectable bleed-through was seen with single-labeled controls. Images were then separated into individual channels by Metamorph imaging software (Universal Imaging, Downingtown, PA). Images containing GluR1 surface puncta were thresholded by gray value at a level close to $50 \%$ of the dynamic range of the acquisition device (i.e., 33,000 for a 16 bit image). Each GluR1 punctum from either a GFPSAP97-expressing construct or an untransfected neighboring neuron was treated as a region, and the characteristics of each punctum, such as pixel area, average fluorescence, and total fluorescence, were logged to a spreadsheet. For spine analysis, neurons were thresholded on the basis of their GFP signal, and spines were outlined manually and then measured by morphometry. Large spines were considered to be $>1$ SD above the mean spine size for neurons expressing only GFP.

Cell culture and neuronal transfection. High-density cortical cultures from 18-d-old embryonic rats were prepared as reported previously (Liao et al., 2001). Briefly, cortices from rat embryos were dissociated by papain and plated onto poly-L-lysine-coated $18 \mathrm{~mm}$ coverslips. Usually, $6 \times 10^{6}$ neurons were added to a $60 \mathrm{~mm}$ culture dish. Growth media contained MEM supplemented with 5\% horse serum and glutamine. Neurons were fed twice per week with glial conditioned growth medium.

Medium-density hippocampal neurons were prepared from postnatal day 0 (P0) to P2 rat pups. Hippocampi were dissociated with $0.25 \%$ trypsin and then plated onto $18 \mathrm{~mm}$ coverslips previously cultured with a confluent bed of glia. Two hippocampi per 12 well plate yielded an ideal density for surface GluR1 quantification experiments. Neurons were plated in MEM containing 5\% heat-inactivated equine serum (Hyclone, Logan, UT), 2\% B27 supplement, 1\% glutamine, and 1\% penicillin/ streptomycin. Cultures were fed once per week.

Low-density hippocampal neurons were prepared as described previously (Banker and Cowan, 1977).

Neuronal transfections were performed either with Lipofectamine 2000 or with the sindbis expression system (Invitrogen, Carlsbad, CA). Double transfections were always performed with Lipofectamine 2000. Sindbis virus was generated by excising enhanced GFP (eGFP), GFPSAP97, or GFP-SAP97 deletions from the pEGFP-C1 vector and subcloning these fragments into the $\mathrm{pSinRep} 5$ vector. The $\mathrm{pSinRep} 5$ vector was then linearized, and RNA transcripts were generated. This RNA and RNA transcripts encoding for viral packaging and replication factors were mixed, electroporated into $\mathrm{BHK}$ cells, and allowed to grow at $37^{\circ} \mathrm{C}$ for $36 \mathrm{hr}$. The medium containing the virus was collected and subsequently centrifuged to concentrate the virus particles. Once the virus was concentrated, the titer was determined empirically by incubating various dilutions of virus with neurons. Usually dilutions of 1:100-1:1000 were sufficient for acceptable transfection efficiency. For both Lipofectamine 2000 and sindbis transfections, the neurons were analyzed $\sim 24 \mathrm{hr}$ after initial incubation.

Cloning and reverse transcription-PCR. GFP-tagged SAP97 constructs, including all deletion mutants, have been described previously (Wu et al., 1998). In this study, all GFP-SAP97 constructs contained the I3 insert unless otherwise noted. In addition, we generated an N-terminal myctagged SAP97 construct by PCR using GFP-SAP97 as a template. The product of this reaction was subcloned into the SalI and NotI restriction sites of pRK5 engineered to contain a single c-myc tag upstream of the SalI site. The resultant clone was sequenced and subsequently expressed in non-neuronal cells and Western blotted to ensure immunoreactivity and correct protein migration.

Total RNA was extracted from whole rat cerebellum or DIV14cultured cortical neurons with Triazol reagents (Invitrogen) according to manufacturer's protocol. Primers were designed from rat SAP-97 mRNA sequence deduced from EST database searches. First-strand cDNA synthesis was performed with Superscript II (Invitrogen) according to the manufacturer's instructions using $5 \mu \mathrm{g}$ of total RNA and a gene-specific primer (SAP-97 GSP AAGAAAGAGCAACATCTGTC) directed against the 3' UTR of rat SAP-97. Thirty-five cycles of PCR were performed with $1 \mu \mathrm{l}$ of first-strand cDNA per $25 \mu \mathrm{l}$ of reaction using Platinum Taq polymerase (Invitrogen) and the following primers: $\mathrm{SH}$, AGATGGTGAGAGTGACGAAGTTGGAGTA; I2, CTTCAGGCCTTTTGATCCCAT-GTC; I3, CTGCTCACTCTGGTCCTTGTTCTTGTAG; I5, GTAACTACTTTCGCTATCGCTGGCATTA. PCR products were analyzed by agarose gel electrophoresis. 
A

$A_{1}$

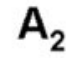

$\mathrm{A}_{3}$

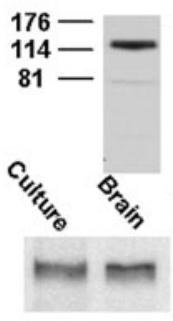

B

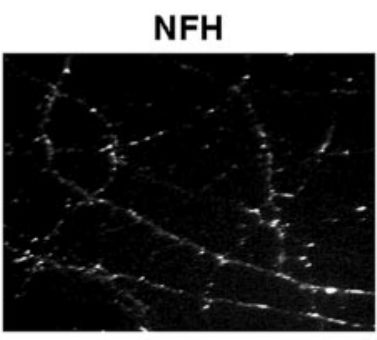

C

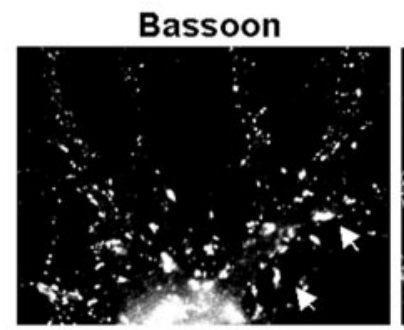

D
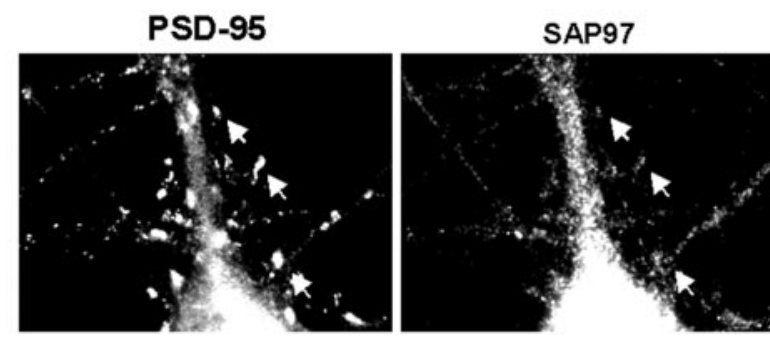

Figure 1. Subcellular localization of endogenous SAP97 in primary hippocampal neurons. A1, Top, Western blot of $\mathrm{p} 2$ fraction from rat forebrain probed with SAP97 polyclonal antibodies raised against a fusion protein corresponding to residues 1-97 of SAP97. Bottom, Western blot showing expression of SAP97 from DIV14 cortical culture lysate (20 $\mu \mathrm{g}$ total protein) or whole rat forebrain lysate (20 $\mu \mathrm{g}$ total protein). $A 2, A 3$, A single hippocampal neuron stained with antibodies against SAP97. The neuron was visualized with a fluorescent microscope at total magnification of $1000 \times . A 3$ is a higher-resolution view of the region from $A 2$. Scale bar, 10 $\mu \mathrm{m}$. B-D, Low-density hippocampal neurons were stained for endogenous SAP97 and either NFH, Bassoon, or PSD-95 monoclonal antibodies. Arrows represent synaptic SAP97 signal.

\section{Results}

\section{Subcellular distribution of SAP97 in dissociated}

hippocampal neurons

We generated polyclonal antibodies to SAP97 (JH 4089) by constructing a fusion protein of residues 1-97 of the published rat SAP97 sequence (Muller et al., 1995). These antibodies recognized a single prominent band from rat forebrain $\mathrm{p} 2$ fractions corresponding to the approximate size of SAP97 published from other studies (Fig. 1A1) (Muller et al., 1995). Furthermore, to confirm SAP97 expression in our culture system, we compared immunoreactivity from rat forebrain with our culture extracts.
We observed similar expression levels of SAP97 in vivo and in vitro (Fig. 1A1). Immunocytochemical labeling of SAP97 revealed an obvious somatodendritic staining pattern in hippocampal neurons (Fig. 1A2-A3). SAP97 immunoreactivity was much higher in the soma than the proximal and distal dendrites. Careful examination of dendritic SAP97 staining revealed a combination of diffuse and a punctate signal (Fig. 1 A3). Immunoreactivity was also seen faintly in distal regions of neurons that followed a distinct pattern but did not appear dendritic (Fig. $1 A 3, B)$. These faint puncta were not random, suggesting that this was not background or nonspecific staining, and were probably axonal in nature. To confirm this in primary culture, we compared the distribution of SAP97 versus the axonal marker neurofilament $\mathrm{H}$ (NFH). NFH clearly labeled axonal projections in our cultures (Fig. 1 B). SAP97 colocalized well with NFH, especially in regions in which SAP97 signal was faint, usually a region with few or no dendrites. This confirms that SAP97 is expressed throughout axons in hippocampal primary culture. Furthermore, we also labeled neurons with an endoplasmic reticulum (ER)-specific marker, BiP (Sans et al., 2001). SAP97 colocalized with BiP, primarily in proximal dendrites, and this colocalization was limited to the interior portion of dendritic shafts (data not shown). To investigate whether endogenous SAP97 is localized to synaptic regions, we labeled neurons with SAP97 in combination with various synaptic markers. To label synaptic terminals, we used primary antibodies against Bassoon, a presynaptic active zone protein (tom Dieck et al., 1998). In DIV21 hippocampal neurons, Bassoon exhibited a punctate signal along the dendritic shaft also in regions that are probably spine synapses (Fig. 1C). SAP97 colocalized with Bassoon in both shaft and spine synapses, suggesting that it is not strictly sequestered to the ER compartment of primary hippocampal neurons, as suggested previously (Sans et al., 2001). There were regions in which Bassoon and SAP97 did not colocalize. This was not surprising, considering the partial ER localization of SAP97 and the fact that Bassoon should label both excitatory and inhibitory synapses (Richter et al., 1999).

To confirm that endogenous SAP97 is present at excitatory synapses, we double-labeled neurons with SAP97 and several postsynaptic protein markers. SAP97 colocalized with PSD-95 in the proximal dendritic shaft and in dendritic spines (Fig. 1D). The SAP97 signal was of a greater intensity in the shaft, but weaker signals were clearly seen in what are probably PSD-95 puncta present in dendritic spines. In addition, SAP97 immunofluorescence overlapped with NR1, further confirming its presence in the postsynaptic density (data not shown).

\section{Synaptic localization of GFP-SAP97 in primary}

cortical neurons

Our data on the spatial distribution of SAP97 in hippocampal neurons indicate that SAP97 is broadly expressed, being present in axons and synapses and with ER-like structures in dendrites. Although these data are consistent with previous studies, they raise the issue of how, when, and whether SAP97 is involved in AMPA receptor trafficking and synaptic function. To begin to address these issues, we examined the spatial distribution of recombinant GFP-tagged SAP97 transfected into either lowdensity hippocampal or high-density cortical neurons. In both types of neurons, GFP-SAP97 was found to be localized in the cell soma and to dendrites and axons (Fig. 2A1,A2). The distribution of GFP-SAP97 in the soma was similar to endogenous SAP97, as shown by colocalization with BiP (Fig. 2A3). However, GFPSAP97 signal along dendritic shafts exhibited a pronounced punctate pattern in both low- and high-density hippocampal 

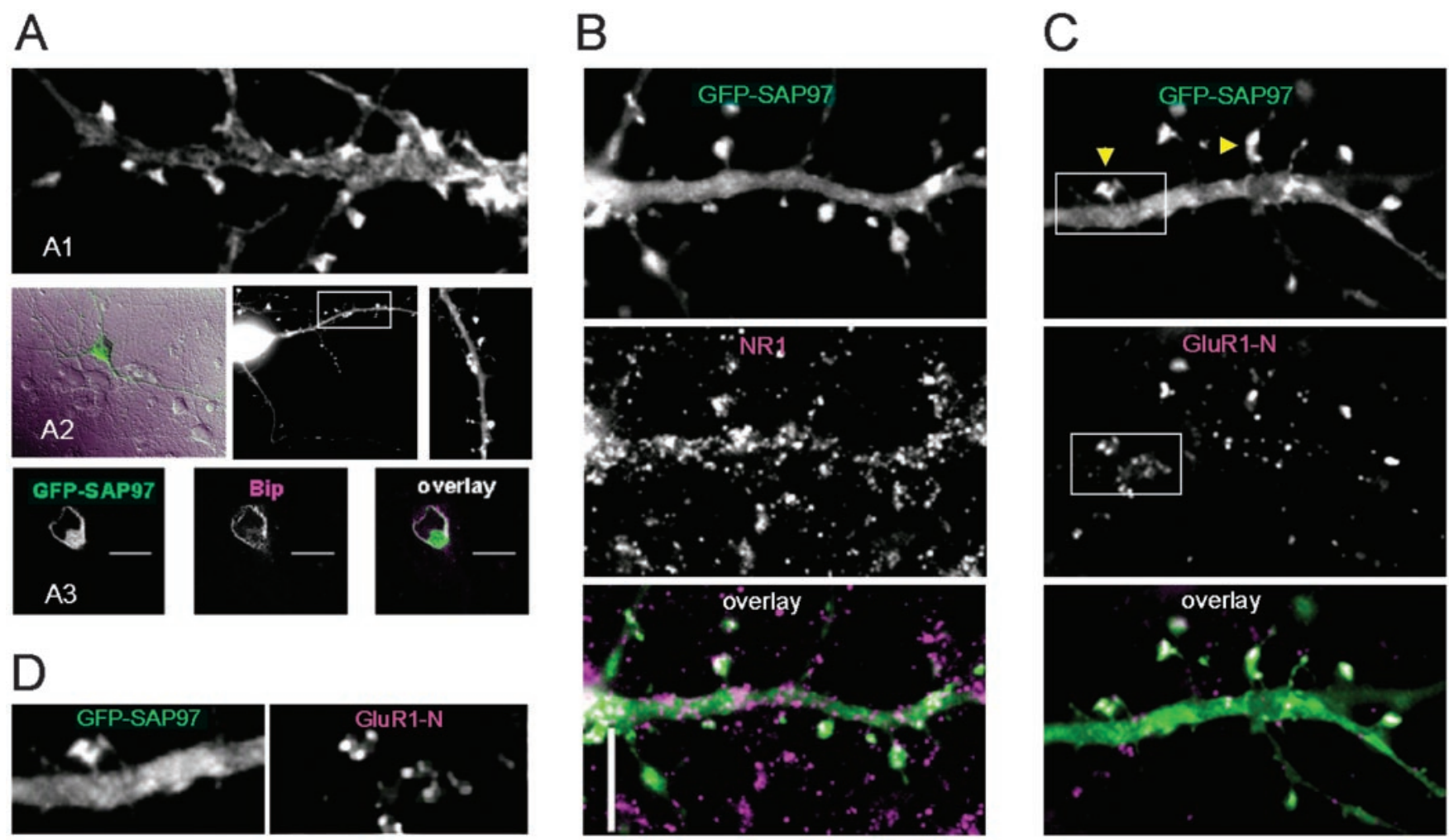

Figure 2. Localization of GFP-SAP97 in primary cultured neurons. A1, A low-density hippocampal neuron was transfected with GFP-SAP97 and subsequently stained with a monoclonal FITC-conjugated anti-GFP antibody. A2, Left, A DIC image with a fluorescence overlay from a DIV14 cortical neuron expressing GFP-SAP97. Middle, The raw GFP signal illustrating the subcellular localization of GFP-SAP97 from a different neuron. Right, A higher-resolution image of the dendrite shown in the middle. A3, A DIV14 cortical neuron expressing GFP-SAP97. Middle, Endogenous BiP signal. Right, Colocalization of the GFP and BiP signals. Scale bar, $5 \mu \mathrm{m}$. b. A DIV14 high-density cortical neuron expressing GFP-SAP97. Middle, The same neuron stained with antibodies against endogenous NR1. Bottom, Composite color picture to illustrate colocalization of the two signals. Scale bar, $5 \mu \mathrm{m}$. C, A DIV14 high-density cortical neuron expressing GFP-SAP97 stained with N-terminal GluR1 antibodies (GluR1-N) to label surface AMPA receptors. Bottom, Colocalization of the two signals. Arrows point to irregularly shaped spines containing both GFP-SAP97 and GluR1. $D$, Boxed regions from C are shown magnified.

neurons (Fig. 2A1,A2) that resembles "spine-like" structures. We quantified the spine versus dendritic shaft signal of GFP-SAP97 by measuring the relative fluorescence intensity of the two structures. This analysis revealed that the fluorescent signal in these spine-like structures was higher than in the adjacent dendrites (see Fig. 5D, below).

To confirm that these structures are indeed dendritic spines, we immunolabeled neurons expressing GFP-SAP97 with glutamate receptor antibodies. Immunostaining high-density cortical neurons with antibodies against the NR1 subunit of NMDA receptors labeled the soma, proximal dendrites, and many fine clusters adjacent to proximal and distal dendrites (Fig. $2 \mathrm{~B}$ ). NR1 puncta often colocalized with these spine-like structures that contained a high GFP-SAP97 signal (Fig. 2B). Furthermore, GluR1containing surface AMPA receptors were often seen in spine-like structures positive for GFP-SAP97 (Fig. 2C). Intriguingly, these clusters appeared larger than surrounding GluR1 clusters from dendrites of neighboring neurons, and multiple GluR1 puncta were often associated with a single spine head (Fig. 2D).

\section{Overexpression of SAP97 causes the enlargement of dendritic spines.}

These results suggested that dendritic morphology might be altered by overexpression of GFP-SAP97. Indeed, spines positive for GFP-SAP97 appeared large and often had irregular shapes (Fig. 2C). To understand the effect that SAP97 may have on dendritic spine morphology, we double-transfected a myc-tagged SAP97 construct together with eGFP and subsequently visualized dendritic morphology through eGFP fluorescence. Double transfections allowed us to measure eGFP signals in neurons expressing myc-SAP97 compared with a neuron transfected with an empty myc vector. To ensure that myc-SAP97 was targeted similarly to GFP-SAP97, neurons were labeled with antibodies against c-myc. Qualitatively, myc-SAP97 signal appeared identical to the distribution pattern of GFP-SAP97, and this signal overlapped nicely with eGFP (Fig. $3 A$ ). Imaging neurons expressing eGFP with or without myc-SAP97 allowed us to accurately visualize the effect this construct had on spine morphology. Neurons expressing only eGFP had many spines but were often small (Fig. 3B). Neurons expressing both myc-SAP97 and eGFP contained a mixture of small and large, irregularly shaped spines, often with two heads sprouting from a single neck (Fig. $3 B$ ). Quantitative analysis of spine morphology revealed a significant increase in the size of the spine head from neurons expressing myc-SAP97 (42.7 \pm 1.9 pixels) compared with neurons expressing only eGFP ( $36.6 \pm 1.4$ pixels; $p<0.05)$. Also, the frequency of large spines (see Materials and Methods) increased by approximately twofold in neurons expressing myc-SAP97 (21.6\%) compared with neurons expressing only eGFP (12.6\%). However, the total number of spines was not different in neurons expressing myc-SAP97 (222 spines; 15 neurons; 3 transfections) compared with neurons expressing only eGFP (293 spines; 18 neurons; 3 transfections). Together, these results indicate that SAP97 is broadly expressed in neurons, and it not only can become concentrated in dendritic spines but also can cause enlargement of the spine head. 
A
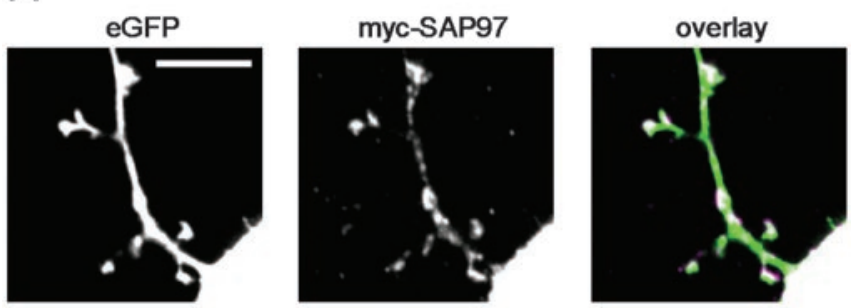

B
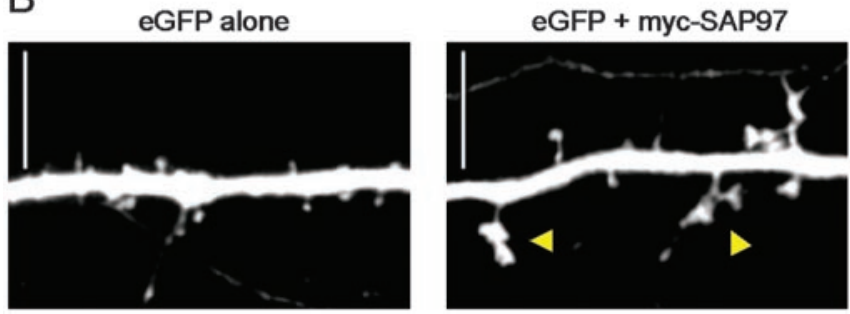

Figure 3. Overexpression of SAP97 causes the enlargement of dendritic spines. A, DIV15 cortical neuron double-transfected with eGFP and myc-SAP97. Right, Overlay of the two signals. Scale bar, $5 \mu \mathrm{m}$. B, Left, GFP signal from a neuron transfected only with eGFP CDNA. Right, GFP signal from a neuron transfected with both eGFP and myc-SAP97 CDNAs. Arrows represent large, irregularly shaped spines. Scale bar, $5 \mu \mathrm{m}$.

Expression of GFP-SAP97 enhances surface expression of AMPA receptors and synaptic transmission

Enlargement of dendritic spines has been shown to correlate with an increase in surface glutamate receptors (El-Husseini et al., 2000a; Sala et al., 2001), and SAP97 has been shown to bind to the C-terminal PDZ ligand of GluR1 in neurons (Leonard et al., 1998). We were therefore interested to investigate the effect of GFP-SAP97 on the surface/synaptic expression of endogenous GluR1-containing AMPA receptors. In these experiments, medium-density hippocampal neurons grown for 10-14 d in vitro and transfected with GFP-SAP97 were labeled live with N-terminal GluR1 antibodies to identify surface AMPA receptors. Hippocampal neurons positive for GFP-SAP97 ( $n=2885$ puncta; 20 neurons; 3 transfections) exhibited a noticeable increase in GluR1 surface staining relative to neighboring untransfected cells ( $n=2755$ puncta; 20 neurons; 3 transfections) (Fig $4 A$ ). Interestingly, regions with intense labeling for anti-GFP also contained unusually intense anti-GluR1 signal. Quantification of surface GluR1-containing AMPA receptors revealed a shift in the distribution of the size of the clusters as shown by the cumulative probability plot (Fig. 4B). Furthermore, when all clusters measured from GFP-SAP97-expressing neurons were averaged and compared with clusters from neighboring untransfected neurons, there was a $26 \%$ increase in the size of GluR1 clusters (Fig. $4 B$ ). Also, there was a small but significant increase in the intensity of the GluR1 puncta (data not shown).

To further investigate the effect GFP-SAP97 has on excitatory synapses, we overexpressed this construct in DIV14-DIV16 cortical neurons and performed mEPSC analysis by whole-cell patch-clamp recording. Neurons expressing GFP-SAP97 exhibited a strong enhancement in the frequency of mEPSCs (Fig. 4C) while showing a slight but nonsignificant decrease in mEPSC amplitude compared with neurons expressing eGFP [frequency: GFP, $0.733 \pm 0.12 \mathrm{~Hz}(n=18)$; GFP-SAP97, $2.68 \pm 0.28 \mathrm{~Hz}(n=$ $18), p<0.0005$; amplitude: GFP, $12.8 \pm 0.72 \mathrm{pA}(n=18)$; GFP-SAP97, $11.4 \pm 0.88 \mathrm{pA}(n=18), p=0.11]$. Other properties of mEPSCs such as rise (GFP, $1.93 \pm 0.72 \mathrm{msec}$; GFP-SAP97, $1.89 \pm 0.53 \mathrm{msec})$ and decay times (GFP, $1.94 \pm 0.18 \mathrm{msec}$; GFP-SAP97, $1.89 \pm 0.21 \mathrm{msec}$ ) were identical between the two groups. In addition, the passive membrane properties of neurons such as $R s$ and $R i$ were not statistically different between neurons expressing eGFP or GFP-SAP97.

GluR1-containing AMPA receptors have been associated with
A
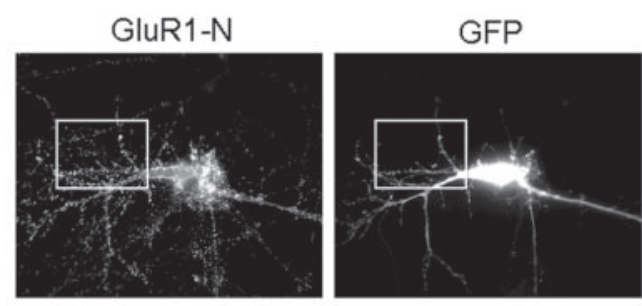

GluR1-N

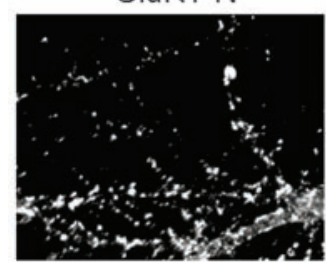

B
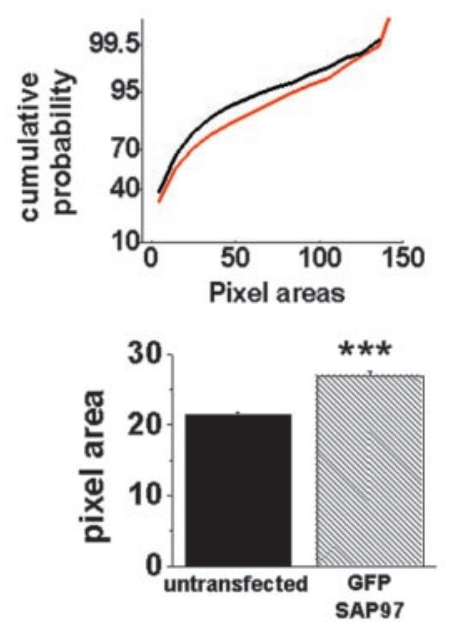

C

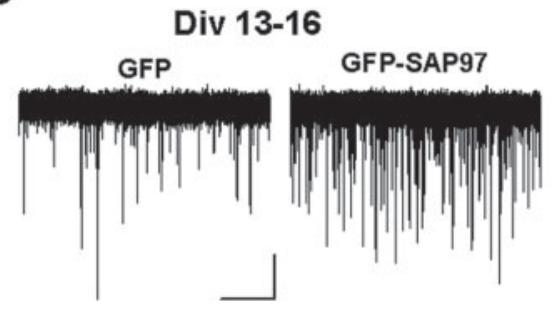

D

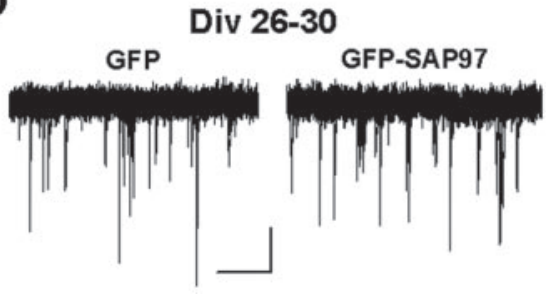

Figure 4. Expression of GFP-SAP97 enhances expression of surface AMPA receptors and synaptic transmission in primary cultured neurons. A, DIV10 medium-density hippocampal neurons were transfected with GFP-SAP97 and subsequently labeled with GluR1-N antibodies. Two neurons are contained in the field shown in A. One neuron is positive for GFP-SAP97, and the other is not. The bottom panels are enlarged versions of the boxed regions in the top panels. B, Quantification of the experiment shown in A. Top, Cumulative probability plot of all GluR1 clusters acquired from GFP-SAP97 (red) or untransfected (black) neurons. Bottom, The average cluster size (in pixels) of all GluR1 puncta from both experimental groups. ${ }^{* *} p<0.0005$. C, DIV13-DIV16 high-density cortical neurons were transfected with GFP or GFP-SAP97. Left traces are from a neuron transfected with GFP, and right traces are from a neuron transfected with GFP-SAP97. In each case, five 5 sec traces 20 sec apart were overlayed to give a more faithful representation of the true mEPSC frequency from these neurons. Calibration: 10 pA, $1 \mathrm{sec}$. See Results for quantification. D, DIV26 -DIV30 high-density cortical neurons were transfected with GFP or GFP-SAP97. Left traces are from a neuron transfected with GFP, and the right traces are from a neuron transfected with GFP-SAP97. In each case, five 5 sec traces taken 20 sec apart were overlayed to give a more faithful representation of the true mEPSC frequency from these neurons. Calibration: $10 \mathrm{pA}, 200 \mathrm{msec}$. See Results for quantification. 
mature neurons or recently activated synapses (Hayashi et al., 2000). Therefore, we wondered what effect GFP-SAP97 would have in older cortical cultures, a time in development at which synapses are more likely to express GluR1-containing AMPA receptors on the surface. mEPSC frequency and amplitude from DIV28 neurons expressing GFP-SAP97 showed no significant difference from cells expressing eGFP (Fig. 4D). There was a trend toward an increase in mEPSC frequency, but it did not reach a level of significance [frequency: GFP, $3.76 \pm 0.85 \mathrm{~Hz}(n=9)$; GFP-SAP97, $5.05 \pm 0.95 \mathrm{~Hz}(n=10), p=$ 0.29; amplitude: GFP, $12.1 \pm 0.92 \mathrm{pA}$ $(n=9)$; GFP-SAP97, $11.5 \pm 1.7 \mathrm{pA}(n=$ $10), p=0.75]$. As in experiments performed with younger cortical neurons, both groups had identical passive properties and no significant differences in rise or decay times of mEPSC events. Finally, we performed experiments comparing eGFP-expressing neurons with untransfected neighbors to control for virus infection in DIV28 primary cortical neurons. Compared with untransfected neighboring neurons, eGFP-expressing neurons had no significant differences in frequency (untransfected, $4.64 \pm 1.1 \mathrm{~Hz}$; eGFP, $4.52 \pm 1.1 \mathrm{~Hz} ; n=8$ for each group) or amplitude (untransfected, $14.1 \pm 1.9 \mathrm{pA}$; eGFP, $12.0 \pm 1.3 \mathrm{pA} ; n=8$ for each group) of miniature events. In addition, passive properties, including $R s$ and $R i$, and rise and decay times of AMPA-mediated mEPSCs, were also not changed. This suggests that virus expression alone does not alter synaptic transmission in cultured neurons.

\section{A protein 4.1 binding motif in SAP97 is} necessary for targeting of GFP-SAP97 to spines

Initially, the targeting of GFP-SAP97 into spines was surprising. In particular, it was unclear why the overexpressed protein seemed to concentrate in dendritic spines, whereas endogenous SAP97 appeared to be more homogeneously distributed throughout neurons. The ability of myc-tagged SAP97 to efficiently localize to synapses, similar to GFP-tagged SAP97, suggests that this is not caused by the tag or overexpression but perhaps by intrinsic sequence elements in SAP97 that, similar to PSD95/SAP90 (Craven et al., 1999), guide it to the synapse. In the case of PSD95/SAP90, palmitoylation of N-terminal cysteine residues has been shown to be important for postsynaptic targeting (Craven et al., 1999; Firestein et al., 2000). The inability of SAP97 to be palmitoylated suggest that different cues may guide this SAP to the synapse (El-Husseini et al., 2000b). On the basis of studies of SAP97 localization in epithelial cells (Wu et al., 1998), there are several regions that could be important for synaptic targeting. We therefore examined the spatial distribution of several GFP-tagged SAP97 deletion constructs transfected into cortical neurons (Fig. $5 A, B)$. Similar to epithelial cells, deleting the SH3 or GUK domains had little effect on the spine localization of SAP97, whereas deleting the hook region $(\Delta \mathrm{I} 3)$ or expressing only the three PDZ domains (PDZ123) leads to a reduced efficacy of targeting SAP97 to the spine head (Fig. 5B-D). In fact, only GFP-SAP97 $\Delta \mathrm{I} 3$ was not significantly different from GFP in respect to spine targeting from all SAP97 mutants quantified (Fig. 5C). The GFP signal in these later two constructs was distributed homogeneously throughout the dendritic tree. These data indicate that although the core domains in SAP97 are not critical for its targeting to dendritic spines, the hook region is a critical element. These observations are consistent with those on SAP97 targeting to the epithelial lateral membrane ( $\mathrm{Wu}$ et al., 1998) and Dlg to the Drosophila neuromuscular junction (Thomas et al., 2000).

As discussed above, the hook region in SAP97 is a site of alternative splicing. To date, four short sequence elements, in various combinations, have been found to be inserted at this site between the SH3 and GUK domains in SAP97 (Lue et al., 1994; Muller et al., 1995; McLaughlin et al., 2002). Although the function of these inserts is generally not known, the $\mathrm{I} 3$ insert, present in the SAP97 constructs used thus far, has been found to bind the actin/spectrin binding protein, protein 4.1 (Lue et al., 1994). The dramatic loss in spine localization of SAP97 $\Delta \mathrm{I} 3$ suggests that 4.1 
A

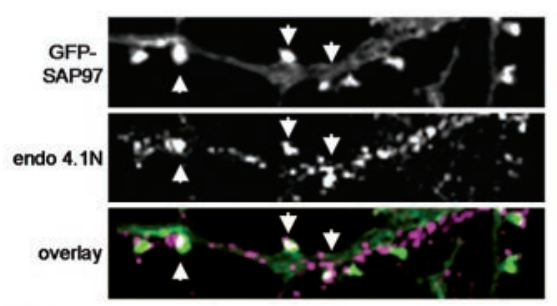

B

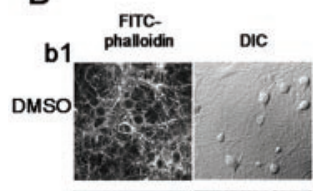

Lat A
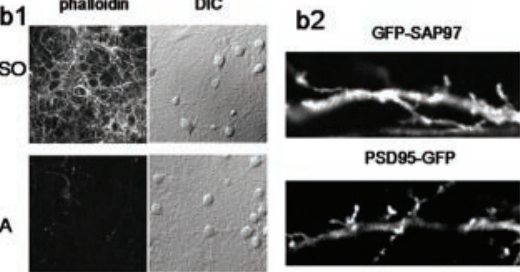

C

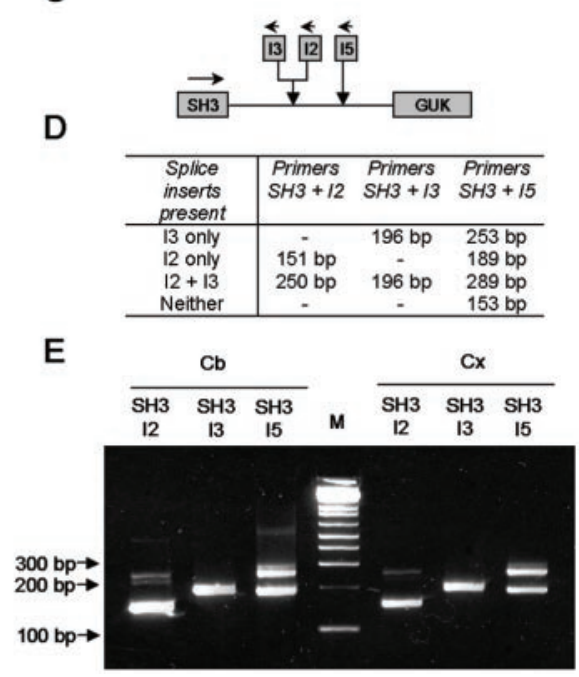

Figure 6. The actin cytoskeleton is essential for the retention of SAP97 in dendritic spines. A, DIV21 low-density hippocampal neuron expressing GFP-SAP97 was labeled with $4.1 \mathrm{~N}$ polyclonal antibodies. The arrows represent areas of intense $4.1 \mathrm{~N}$ immunoreactivity that colocalize with spine-enriched GFP-SAP97. B, DIC or fluorescent images from neurons treated with DMS0 or $5 \mu \mathrm{M}$ latrunculin A. After latrunculin A treatment, both sets of neurons were then labeled with FITC-conjugated phalloidin. Fluorescent images in 61 were acquired with a confocal microscope with identical detection settings. In addition, both images were also scaled equally to show the true difference in FITC signal. b2, Selected dendrite from a neuron expressing either GFP-SAP97 or PSD95-GFP in the presence of $5 \mu \mathrm{m}$ latrunculin A. C, Schematic representing location of spliced inserts between the SH3 and GK domains. Arrows represent primers used in RT-PCR. D, Table indicating predicted sizes of RT-PCR products produced from each primer sets shown in D.E, RT-PCRs from either whole cerebellum (cb) or DIV14 cortical culture (cx).

binding to SAP97 is critical for synaptic targeting. To confirm this finding and ensure that deleting these residues does not exert a nonspecific effect on SAP97 structure, we transfected a GFPtagged SAP97 isoform containing the I2 insert and examined its distribution. Importantly, the I2 insert has been shown previously not to bind protein 4.1 (Lue et al., 1994). The distribution of GFP-SAP97-I2 in neurons was diffuse throughout the soma and dendrites and was not specifically concentrated in spines (Fig. $5 D$ ). In dendrites, the distribution of this splice form was indistinguishable from neurons expressing GFP-SAP97 $\Delta \mathrm{I} 3$ or GFPPDZ123 (Fig. 5B-D). In addition, the I2 construct failed to increase the size of spines in high-density cortical neurons (data not shown), consistent with a central role for synaptic SAP97 isoforms in stimulating changes in synapse/spine size.

The actin cytoskeleton is essential for the retention of SAP97 in dendritic spines

The ability of protein 4.1 to bind the I3 domain of SAP97 (Lue et al., 1994) and the presence of the neuronal isoform of protein 4.1 (4.1N) at synapses (Walensky et al., 1999) suggest that the actin/ spectrin binding activity of protein 4.1 isoforms would be essential for the spine localization of SAP97 isoforms containing an I3 insert. Indeed, we found that GFP-SAP97 colocalizes well with endogenous $4.1 \mathrm{~N}$ from hippocampal neurons, especially at regions that are probably synapses (Fig. $6 A$ ). Therefore, we hypothesized that disruption of the actin cytoskeleton with latrunculin A, a treatment known to disrupt SAP97 targeting in epithelial cells (Reuver and Garner, 1998), would reduce the spine targeting efficiency of GFP-SAP97 in neurons. In cortical neurons treated with latrunculin A for $9 \mathrm{hr}$, we observed a severe disruption of the F-actin signal (Fig. 6 Bb1), whereas no qualitative change in neuronal morphology or cell viability was observed (Fig. 6Bb1). To study the possible effects of F-actin disruption on GFP-SAP97 targeting, we transfected high-density cortical neurons with
GFP-SAP97or PSD95-GFP and observed the GFP signal. PSD-95-GFP was used as a control for synaptic targeting because endogenous PSD-95 targeting is usually insensitive to actin disruption (Allison et al., 1998). As expected, the localization of PSD95-GFP appeared relatively normal after the treatment of our cultures with latrunculin A for $9 \mathrm{hr}$, exhibiting a strong fluorescent signal at spines (Fig. 6Bb2). Note, however, that spines appeared smaller compared with neurons treated with DMSO only. In contrast, latrunculin A treatment of neurons expressing GFPSAP97 resulted in a diffuse fluorescent signal. In fact, it was difficult to locate any neurons with a spine-enriched phenotype common to GFP-SAP97 expression in neurons, and GFP-SAP97 from neurons treated with latrunculin A resembled neurons expressing GFP-SAP97 $\Delta \mathrm{I} 3$ or GFP-SAP97I2.

On the basis of these results, the I3 region of SAP97 may be important for synaptic targeting of this protein in primary culture. However, expression of this spliced insert in our culture system must be confirmed. To confirm the existence of the I3 isoform of SAP97 in cultured cortical neurons, we performed reverse transcription (RT)-PCR from DIV14 culture lysates. Primers were designed against the $3^{\prime}$ end of the $\mathrm{SH} 3$ sequence and either the I2, I3, or I5 sequence (Fig. 6C). RT-PCR results confirmed the existence of I2, I3, and I5 splice variants of SAP97 in cortical culture (Fig. 6E). Our RT-PCR data from primary culture are in agreement with previous data showing the existence of the I3 isoform of SAP97 identified from a human brain cDNA library (McLaughlin et al., 2002).

GFP-SAP97-enhanced AMPA receptor surface expression and synaptic transmission are dependent on the $\mathrm{I} 3$ sequence

To investigate a potential role of the I3 sequence in AMPA receptor surface expression, we transfected neurons with GFPSAP97 $\Delta \mathrm{I} 3$ and subsequently labeled neurons with GluR1-N antibodies. Neurons expressing GFP-SAP97 $\Delta \mathrm{I} 3$ stained positive for GluR1, but the signal was of similar intensity or slightly less than in neighboring neurons (Fig. 7A). All clusters from neurons expressing SAP97 $\Delta \mathrm{I} 3$ and untransfected neighbors were binned by pixel area, and their cumulative probability plots were superimposed (Fig. 7B). Unlike GFP-SAP97-transfected neurons, clusters from GFPSAP97 $\mathrm{I} 3$ did not shift to larger values compared with untransfected controls. Furthermore, when all clusters from GFP-SAP97 $\Delta \mathrm{I} 3$ ( $n=1827 ; 19$ neurons; 3 transfections) were compared with clusters from neighboring untransfected neurons ( $n=2533 ; 20$ neurons; 3 transfections) and averaged, there was actually a slight but significant decrease in cluster size (Fig. 7B). Considering these results, we were curious what effect GFPSAP97 $\Delta \mathrm{I} 3$ would have on mEPSCs. Interestingly, the frequency of mEPSCs from neurons expressing GFP-SAP97 $\Delta \mathrm{I} 3$ was not statistically different from that of eGFP-expressing neurons (Fig. $7 C, D)[\mathrm{GFP}, 0.754 \pm 0.14 \mathrm{~Hz}(n=9)$; GFP-SAP97, $0.875 \pm 0.17$ $\mathrm{Hz}(n=9) ; p=0.60]$. Furthermore, the average mEPSC amplitude was also not significantly different compared with eGFPexpressing neurons [GFP, $18.0 \pm 3.0 \mathrm{pA}(n=9)$; GFP-SAP97, 
A
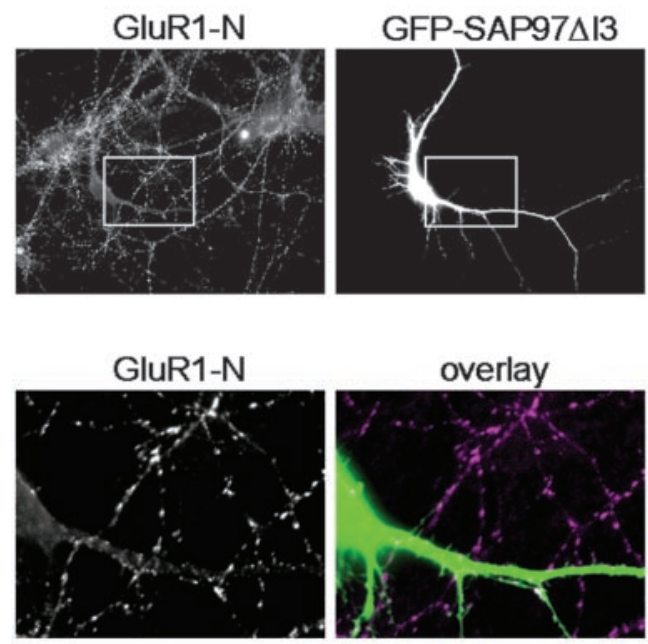

B
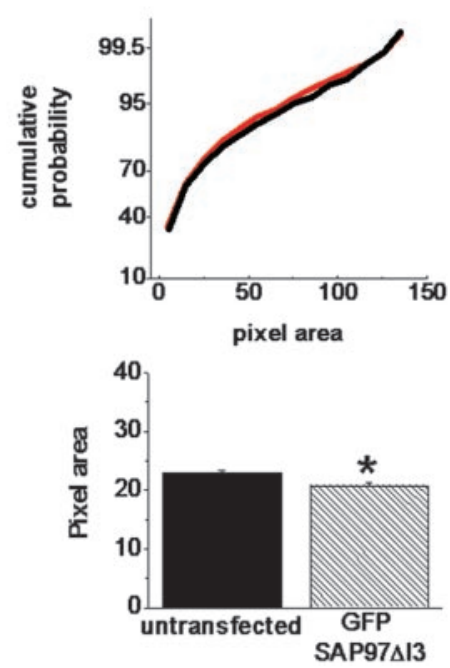

C
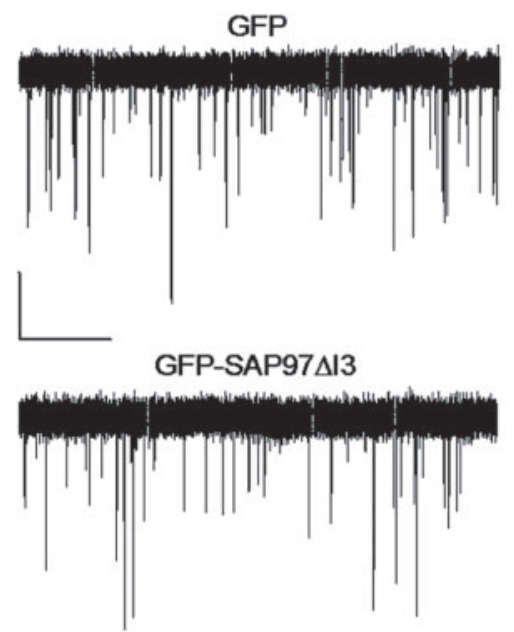

Figure 7. GFP-SAP97 enhancement of synaptic transmission and surface AMPA receptors is dependent on the 13 sequence. $A$, DIV10 medium-density hippocampal neurons were transfected with GFP-SAP97 $\Delta \mathrm{I} 3$ and subsequently labeled with GluR1-N antibodies. Several neurons are contained in the field shown in $A$. One neuron is positive for GFP-SAP97 $\Delta \mathrm{I}$, and the others are not. The bottom panels are enlarged versions of the boxed regions in the top panels. $B$, Quantification of the experiments shown in $A$. Top, Cumulative probability plot of all GluR1 clusters acquired from GFP-SAP97 $\Delta \mathrm{I} 3$ (red) or untransfected (black) neurons. Bottom, Average cluster size (in pixels) of all GluR1 puncta from both experimental groups. * $p<0.05$. C, DIV13-DIV16 high-density cortical neurons were transfected with GFP or GFP-SAP97 $\Delta$ I3. Top traces are from a neuron transfected with GFP-SAP97 $\Delta \mathrm{l} 3$, and bottom traces are from a neuron transfected with GFP. In each case, five 5 sec traces 20 sec apart were overlayed to give a more faithful representation of the true mEPSC frequency from each neuron. Calibration: 10 pA, 1 sec. See Results for quantification.

$19.0 \pm 2.2 \mathrm{pA}(n=9) ; p=0.79]$. All other parameters of mEPSCs were unchanged between the two groups. This suggests that the I3 sequence is necessary for SAP97-mediated increases in surface AMPA receptors and alterations in $\mathrm{mEPSC}$ frequency.

\section{Discussion}

In this study, we describe the subcellular localization of SAP97 in primary hippocampal neurons. SAP97 immunoreactivity appeared to be primarily somatodendritic, although signals in the axon were also detected. Furthermore, we confirmed the presence of SAP97 at excitatory synapses, as indicated by colocalization with NMDA receptors, PSD-95, and Bassoon. This is in contrast to the original characterization of SAP97, because it was reported to be restricted to axons (Muller et al., 1995), and our data are in support of several recent studies showing a predominantly postsynaptic locus of SAP97 expression (Valtschanoff et al., 2000; Aoki et al., 2001). However, in our study, the highest expression of SAP97 appeared in perinuclear regions and in proximal dendrites that contained a high degree of colocalization with ER markers. This is in agreement with a recent study that shows that SAP97 immunoreactivity from primary neurons is not enriched in synapses (Sans et al., 2001). The discrepancy in the distribution pattern of endogenous SAP97 in primary culture versus electron micrographic studies from intact tissue and our data on synaptically enriched recombinant SAP97 (see below) could potentially be a result of the availability of antibody epitopes to SAP97 within the PSD. In addition, intact tissue may contain a higher concentration of SAP97 in the PSD.

Targeting of SAP family proteins in neurons has been well studied, with the exception of SAP97. PSD-93, PSD-95, and SAP102 contain signals within their respective N-terminal domains that are responsible for subcellular targeting in neurons (Firestein et al., 2000). However, in this study, we provide evidence for a novel mechanism of SAP targeting in neurons. We show that targeting of SAP97 in neurons can be splice-form dependent. Constructs of SAP97 containing the I3 spliced insert target preferentially to spines compared with constructs with this region deleted or with a SAP97 isoform that contains an alternatively spliced cassette. Previous studies have indicated that expressed SAP97 in neurons is localized diffusely, whereas other SAP family members are concentrated at excitatory synapses (Craven et al., 1999; Firestein et al., 2000). It is unclear what splice form of SAP97 was used in these overexpression studies, but considering our findings, this may be one explanation for this apparent discrepancy. This finding may also prove relevant for targeting of other SAP proteins. PSD-93/Chapsyn-110 and SAP102 also contain an alternatively spliced region between their SH3 and GK domains (Kim et al., 1996; Muller et al., 1996). In addition, Chapsyn-110 contains an insert that is very similar to the I3 sequence of SAP97. It is possible that this sequence may also play a role in targeting of PSD-93/Chapsyn-110 in neurons.

SAP97 is also expressed in epithelial cells, in which it is actively targeted to sites of cell-cell contact (Reuver and Garner, 1998; Wu et al., 1998). This targeting is dependent on the actin cytoskeleton, because disruption of F-actin results in a diffuse SAP97 signal (Reuver and Garner, 1998). We show that in neurons, the I3 splice form of SAP97 is targeted to excitatory synapses, also a region of cell-cell contact. Furthermore, we show that disruption of the neuronal actin cytoskeleton results in a loss of GFP-SAP97 synaptic targeting, although with little effect on PSD-95-GFP localization. This suggests that SAP97 targeting in neurons is also regulated by binding to the actin cytoskeleton. A neuronal homolog of protein 4.1 has been described previously (Walensky et al., 1999). Protein $4.1 \mathrm{~N}$ binds to the actin cytoskeleton and to GluR1 and is present at excitatory synapses in culture (Shen et al., 2000). Considering that SAP97 also binds to GluR1 (Leonard et al., 1998) and is capable of binding 4.1 homologs (Lue et al., 1994), this presents an attractive model for AMPA receptor trafficking at synapses, where a complex of 4.1-SAP97AMPA receptors may coexist. Furthermore, DLG, the Drosophila homolog of SAP proteins, is targeted to septate junctions through 
its hook domain (Hough et al., 1997). The hook domain is a sequence situated between the SH3 and GK domains of DLG and is analogous to the I3 region of SAP97. Our results show that the I3 insert is expressed in cortical culture. This agrees with a recent study reporting both I 2 and I 3 splice cassettes of SAP97 in human brain (McLaughlin et al., 2002), which also supports a possible role of SAP97 splice variants influencing protein targeting and synaptic function. Our findings do not rule out the possibility of other domains regulating subcellular targeting of SAP97 in neurons, however. Indeed, the primary signal responsible for membrane targeting of SAP97 in epithelial cells was contained within amino acids $1-65$ of the $\mathrm{N}$ terminus (Wu et al., 1998). Deletion of the $\mathrm{I} 3$ region in that study only reduced the efficiency of membrane targeting, but the $\mathrm{N}$ terminus alone was sufficient to mediate targeting of SAP97 to sites of cell-cell contact (Wu et al., 1998). This raises the possibility that the $\mathrm{N}$ terminus of SAP97 may also be involved in neuronal targeting. Together, these data suggest that SAPs contain multiple targeting sequences and may act alone or cooperatively to mediate protein targeting.

In this study, we show that GFP-SAP97 targeting to spines results in morphological changes including enlargement of the head combined with spines exhibiting apparent multiple heads sprouting from a single neck. Furthermore, there is an increase in mEPSC frequency in neurons expressing GFP-SAP97 at 2 weeks of age, although this effect disappears from 4-week-old neurons. Recent evidence suggests that as excitatory synapses mature, there is an increased likelihood that they will contain both AMPA and NMDA receptors (Liao et al., 1999; Petralia et al., 1999). In addition, spines from mature neurons are often larger, and a recent study describes the correlation between spine size and AMPA responses (Matsuzaki et al., 2001). Together, it is likely that expression of GFP-SAP97 has a positive effect on the maturation of synapses in vitro. However, as tempting as it is to speculate that the mechanism leading to an increase in surface AMPA receptors and the facilitation of synaptic transmission by GFPSAP97 involves a direct interaction between it and GluR1 near the postsynaptic membrane, our results do not definitively support such a conclusion. It is possible that SAP97 targeting to spines results in some other mechanism that can lead to the observed effects, such as a coordinated maturation of both postsynaptic and presynaptic processes. Indeed, PSD-95-GFP results in enhancement of mEPSC events and enlargement of dendritic spines, although this SAP does not bind directly to AMPA receptors and the mechanism is thought to be both presynaptic and postsynaptic in nature (El-Husseini et al., 2000a). In addition, overexpression of other scaffolding proteins that do not bind to AMPA receptors can also lead to changes in their synaptic localization (Sala et al., 2001).

It is curious that the main effect on mEPSCs was an increase in frequency rather than amplitude, considering the change in surface expression of AMPA receptors after overexpression of GFPSAP97. We speculate that GFP-SAP97 may result in synapse splitting, causing an overall increase in postsynaptic sites. We feel that this is possible because of the unusually large and irregularly shaped spines that result after GFP-SAP97 expression. We often noticed that multiple GluR1 puncta appeared to be colocalized with these large spines. However, because of the limits of light microscopy, precise measurements were not possible. If this phenomenon were true, several closely apposing puncta could be counted as single large puncta during quantification of surface AMPA receptors. Indeed, the major effect on surface AMPARs by GFP-SAP97 was increased cluster size, whereas there was little effect on cluster brightness.
A recent report has shown that various synaptic proteins, including AMPA receptors, have normal subcellular localization in mice expressing a truncated form of SAP97 (Klocker et al., 2002). These results were not unexpected and are not in disagreement with this study, simply because of the likelihood of functional redundancy brought about by expression of multiple SAPs in most neuronal subtypes. In support of this idea, mice expressing a truncated form of PSD-95 have normal AMPA-mediated synaptic transmission and presumably normal postsynaptic AMPAR expression (Migaud et al., 1998), although hippocampal neurons overexpressing PSD-95 have remarkable increases in AMPAmediated synaptic transmission and surface AMPA receptors (ElHusseini et al., 2000a; Schnell et al., 2002). Therefore, we feel that overexpression of SAP97 constructs in primary culture can lead to greater understanding of molecular organization of proteins at synaptic junctions by identifying a critical spliced region responsible for influencing synaptic transmission.

\section{References}

Allison DW, Gelfand VI, Spector I, Craig AM (1998) Role of actin in anchoring postsynaptic receptors in cultured hippocampal neurons: differential attachment of NMDA versus AMPA receptors. J Neurosci 18:2423-2436.

Aoki C, Miko I, Oviedo H, Mikeladze-Dvali T, Alexandre L, Sweeney N, Bredt DS (2001) Electron microscopic immunocytochemical detection of PSD-95, PSD-93, SAP-102, and SAP-97 at postsynaptic, presynaptic, and nonsynaptic sites of adult and neonatal rat visual cortex. Synapse 40:239-257.

Banke TG, Bowie D, Lee H, Huganir RL, Schousboe A, Traynelis SF (2000) Control of GluR1 AMPA receptor function by cAMP-dependent protein kinase. J Neurosci 20:89-102.

Banker GA, Cowan WM (1977) Rat hippocampal neurons in dispersed cell culture. Brain Res 126:397-442.

Brenman JE, Christopherson KS, Craven SE, McGee AW, Bredt DS (1996) Cloning and characterization of postsynaptic density 93, a nitric oxide synthase interacting protein. J Neurosci 16:7407-7415.

Cai C, Coleman SK, Niemi K, Keinanen K (2002) Selective binding of synapse-associated protein 97 to GluR-A alpha-amino-5-hydroxy-3methyl-4-isoxazole propionate receptor subunit is determined by a novel sequence motif. J Biol Chem 277:31484-31490.

Chen L, Chetkovich DM, Petralia RS, Sweeney NT, Kawasaki Y, Wenthold RJ, Bredt DS, Nicoll RA (2000) Stargazing regulates synaptic targeting of AMPA receptors by two distinct mechanisms. Nature 408:936-943.

Cho KO, Hunt CA, Kennedy MB (1992) The rat brain postsynaptic density fraction contains a homolog of the Drosophila discs-large tumor suppressor protein. Neuron 9:929-942.

Colledge M, Dean RA, Scott GK, Langeberg LK, Huganir RL, Scott JD (2000) Targeting of PKA to glutamate receptors through a MAGUK-AKAP complex. Neuron 27:107-119.

Craven SE, El-Husseini AE, Bredt DS (1999) Synaptic targeting of the postsynaptic density protein PSD-95 mediated by lipid and protein motifs. Neuron 22:497-509.

El-Husseini AE, Schnell E, Chetkovich DM, Nicoll RA, Bredt DS (2000a) PSD-95 involvement in maturation of excitatory synapses. Science 290:1364-1368.

El-Husseini AE, Topinka JR, Lehrer-Graiwer JE, Firestein BL, Craven SE, Aoki C, Bredt DS (2000b) Ion channel clustering by membraneassociated guanylate kinases: differential regulation by $\mathrm{N}$-terminal lipid and metal binding motifs. J Biol Chem 275:23904-23910.

Firestein BL, Craven SE, Bredt DS (2000) Postsynaptic targeting of MAGUKs mediated by distinct N-terminal domains. NeuroReport 11:3479-3484.

Garner CC, Nash J, Huganir RL (2000) PDZ domains in synapse assembly and signalling. Trends Cell Biol 10:274-280.

Hayashi Y, Shi SH, Esteban JA, Piccini A, Poncer JC, Malinow R (2000) Driving AMPA receptors into synapses by LTP and CaMKII: requirement for GluR1 and PDZ domain interaction. Science 287:2262-2267.

Hough CD, Woods DF, Park S, Bryant PJ (1997) Organizing a functional junctional complex requires specific domains of the Drosophila MAGUK discs large. Genes Dev 11:3242-3253. 
Kim E, Cho KO, Rothschild A, Sheng M (1996) Heteromultimerization and NMDA receptor-clustering activity of Chapsyn-110, a member of the PSD-95 family of proteins. Neuron 17:103-113.

Kim JH, Liao D, Lau LF, Huganir RL (1998) SynGAP: a synaptic RasGAP that associates with the PSD-95/SAP90 protein family. Neuron 20:683-691.

Kistner U, Wenzel BM, Veh RW, Cases-Langhoff C, Garner AM, Appeltauer U, Voss B, Gundelfinger ED, Garner CC (1993) SAP90, a rat presynaptic protein related to the product of the Drosophila tumor suppressor gene dlg-A. J Biol Chem 268:4580-4583.

Klocker N, Bunn RC, Schnell E, Caruana G, Bernstein A, Nicoll RA, Bredt DS (2002) Synaptic glutamate receptor clustering in mice lacking the SH3 and GK domains of SAP97. Eur J Neurosci 16:1517-1522.

Lee HK, Barbarosie M, Kameyama K, Bear MF, Huganir RL (2000) Regulation of distinct AMPA receptor phosphorylation sites during bidirectional synaptic plasticity. Nature 405:955-959.

Leonard AS, Davare MA, Horne MC, Garner CC, Hell JW (1998) SAP97 is associated with the alpha-amino-3-hydroxy-5-methylisoxazole-4propionic acid receptor GluR1 subunit. J Biol Chem 273:19518-19524.

Liao D, Zhang X, O’Brien R, Ehlers MD, Huganir RL (1999) Regulation of morphological postsynaptic silent synapses in developing hippocampal neurons. Nat Neurosci 2:37-43.

Liao D, Scannevin RH, Huganir R (2001) Activation of silent synapses by rapid activity-dependent synaptic recruitment of AMPA receptors. J Neurosci 21:6008-6017.

Lue RA, Marfatia SM, Branton D, Chishti AH (1994) Cloning and characterization of hdlg: the human homologue of the Drosophila discs large tumor suppressor binds to protein 4.1. Proc Natl Acad Sci USA 91:9818-9822.

Matsuzaki M, Ellis-Davies GC, Nemoto T, Miyashita Y, Iino M, Kasai H (2001) Dendritic spine geometry is critical for AMPA receptor expression in hippocampal CA1 pyramidal neurons. Nat Neurosci 4:1086-1092.

McLaughlin M, Hale R, Ellston D, Gaudet S, Lue RA, Viel A (2002) The distribution and function of alternatively spliced insertions in $\mathrm{hDlg}$. J Biol Chem 277:6406-6412.

Migaud M, Charlesworth P, Dempster M, Webster LC, Watabe AM, Makhinson M, He Y, Ramsay MF, Morris RG, Morrison JH, O'Dell TJ, Grant SG (1998) Enhanced long-term potentiation and impaired learning in mice with mutant postsynaptic density-95 protein. Nature 396:433-439.

Muller BM, Kistner U, Veh RW, Cases-Langhoff C, Becker B, Gundelfinger ED, Garner CC (1995) Molecular characterization and spatial distribution of SAP97, a novel presynaptic protein homologous to SAP90 and the Drosophila discs-large tumor suppressor protein. J Neurosci 15:2354-2366.

Muller BM, Kistner U, Kindler S, Chung WJ, Kuhlendahl S, Fenster SD, Lau LF, Veh RW, Huganir RL, Gundelfinger ED, Garner CC (1996) SAP102, a novel postsynaptic protein that interacts with NMDA receptor complexes in vivo. Neuron 17:255-265.
Petralia RS, Esteban JA, Wang YX, Partridge JG, Zhao HM, Wenthold RJ, Malinow R (1999) Selective acquisition of AMPA receptors over postnatal development suggests a molecular basis for silent synapses. Nat Neurosci 2:31-36.

Reuver SM, Garner CC (1998) E-cadherin mediated cell adhesion recruits SAP97 into the cortical cytoskeleton. J Cell Sci 111:1071-1080.

Richter K, Langnaese K, Kreutz MR, Olias G, Zhai R, Scheich H, Garner CC, Gundelfinger ED (1999) Presynaptic cytomatrix protein bassoon is localized at both excitatory and inhibitory synapses of rat brain. J Comp Neurol 408:437-448.

Sala C, Piech V, Wilson NR, Passafaro M, Liu G, Sheng M (2001) Regulation of dendritic spine morphology and synaptic function by Shank and Homer. Neuron 31:115-130.

Sans N, Racca C, Petralia RS, Wang YX, McCallum J, Wenthold RJ (2001) Synapse-associated protein 97 selectively associates with a subset of AMPA receptors early in their biosynthetic pathway. J Neurosci 21:7506-7516.

Schnell E, Sizemore M, Karimzadegan S, Chen L, Bredt DS, Nicoll RA (2002) Direct interactions between PSD-95 and stargazin control synaptic AMPA receptor number. Proc Natl Acad Sci USA 99:13902-13907.

Shen L, Liang F, Walensky LD, Huganir RL (2000) Regulation of AMPA receptor GluR1 subunit surface expression by a $4.1 \mathrm{~N}$-linked actin cytoskeletal association. J Neurosci 20:7932-7940.

Shi SH, Hayashi Y, Petralia RS, Zaman SH, Wenthold RJ, Svoboda K, Malinow R (1999) Rapid spine delivery and redistribution of AMPA receptors after synaptic NMDA receptor activation. Science 284:1811-1816.

Thomas U, Ebitsch S, Gorczyca M, Koh YH, Hough CD, Woods D, Gundelfinger ED, Budnik V (2000) Synaptic targeting and localization of discs-large is a stepwise process controlled by different domains of the protein. Curr Biol 10:1108-1117.

tom Dieck S, Sanmarti-Vila L, Langnaese K, Richter K, Kindler S, Soyke A, Wex H, Smalla KH, Kampf U, Franzer JT, Stumm M, Garner CC, Gundelfinger ED (1998) Bassoon, a novel zinc-finger CAG/glutaminerepeat protein selectively localized at the active zone of presynaptic nerve terminals. J Cell Biol 142:499-509.

Valtschanoff JG, Burette A, Davare MA, Leonard AS, Hell JW, Weinberg RJ (2000) SAP97 concentrates at the postsynaptic density in cerebral cortex. Eur J Neurosci 12:3605-3614.

Walensky LD, Blackshaw S, Liao D, Watkins CC, Weier HU, Parra M, Huganir RL, Conboy JG, Mohandas N, Snyder SH (1999) A novel neuronenriched homolog of the erythrocyte membrane cytoskeletal protein 4.1. J Neurosci 19:6457-6467.

Wu H, Reuver SM, Kuhlendahl S, Chung WJ, Garner CC (1998) Subcellular targeting and cytoskeletal attachment of SAP97 to the epithelial lateral membrane. J Cell Sci 111:2365-2376. 